\title{
Le cout de production de l'électricité nucléaire
}

Anne-Sophie Dessillons, rapporteure de la Cour des comptes

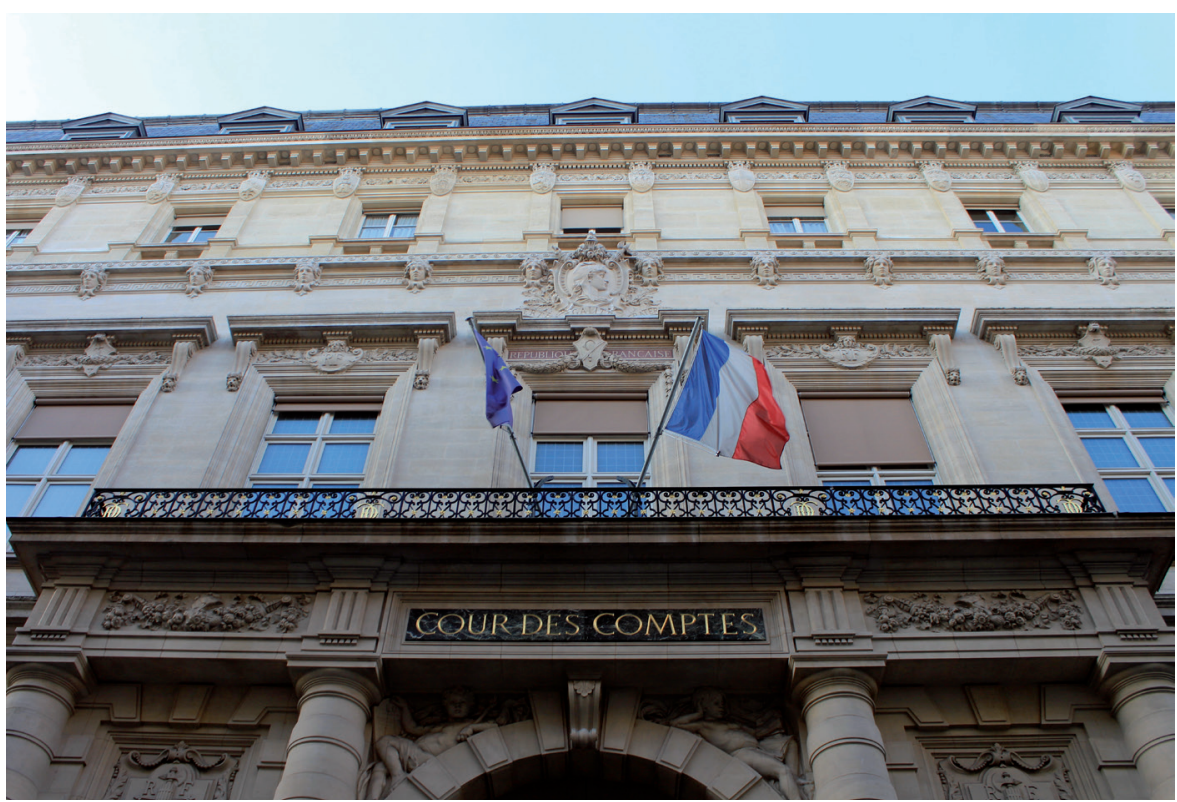

Le cout de production d'un mégawatt-heure (MWh) d'électricité nucléaire est un paramètre essentiel dans l'évaluation

de l'intérêt économique de la filière. La Cour des comptes

a produit un rapport très détaillé qui a fait débat, et dont les éléments chiffrés permettent de se faire une idée plus claire sur les couts d'exploitation (hors recherche et développement) du nucléaire actuel et sur leur évolution dans le futur.

Dans le contexte actuel de craintes croissantes sur la sureté du nucléaire (post-Fukushima, difficultés sur le chantier de Flamanville, fermetures préventives de réacteurs à l'automne $2016 \ldots$ ) et de montée en puissance des énergies renouvelables affichant des baisses de cout importantes, le cout du nucléaire est plus que jamais au centre des débats. Il l'est d'autant plus que des incertitudes pèsent sur son évolution avec les investissements de grand carénage ou encore le financement des réacteurs de $3^{\mathrm{e}}$ génération. La Cour des comptes a eu l'occasion de se pencher sur la question à deux reprises, en 2012 [1] puis en 2014 [2] : ses analyses guideront les propos suivants.

\section{Un cout de production avoisinant les $60 € / \mathrm{MWh}$, en forte augmentation}

La Cour des comptes a estimé le cout de production du parc nucléaire existant à $60 € / \mathrm{MWh}$ en 2013 contre $50 € / \mathrm{MWh}$ en $2010^{\text {(a) }}$. Cette augmentation de 20\% en trois ans trouve une explication dans trois facteurs.

Tout d'abord, les investissements de maintenance constituent le poste de dépenses ayant le plus augmenté : leur progression explique la moitié de la hausse du cout de production. Cette progression est due à trois facteurs :

- un rattrapage du niveau d'investissements «normaux » (21\% des investissements de 


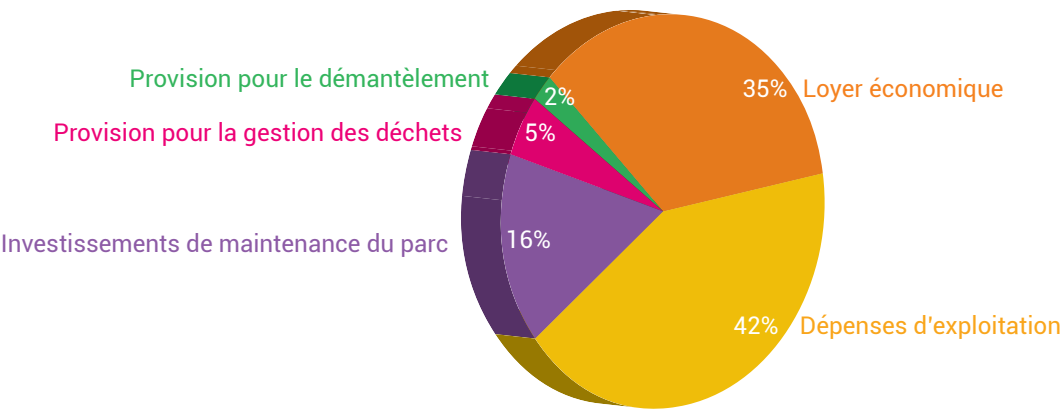

1. Part des différentes contributions au cout de production de l'électricité nucléaire française.

$\gg>$

maintenance), la faiblesse de ces derniers au début des années 2000 ayant eu des répercussions négatives sur l'exploitation et la production ;

- la nécessité de rénovation ou de remplacement de certains gros composants dont la durée de vie est inférieure à 40 ans $(29 \%$ des investissements de maintenance) : il s'agit notamment des générateurs de vapeur, des alternateurs, des condenseurs ou des éléments des tours réfrigérantes ;

- la forte augmentation des investissements de sureté (50\% des investissements de maintenance) dans un contexte postFukushima et pour permettre l'allongement de la durée d'exploitation des réacteurs, l'Autorité de Sûreté Nucléaire (ASN) ayant conditionné cette dernière à une amélioration de la sureté pour les amener au niveau des objectifs de sureté des réacteurs de $3^{e}$ génération.

Ensuite, la forte progression des dépenses d'exploitation $(+31 \%$ en euros courants à périmètre constant) explique quant à elle un quart de la hausse du cout de production. Cette hausse des dépenses d'exploitation trouve une part d'explication dans la hausse des dépenses d'achats et de logistique induite par la progression des investissements de maintenance précédemment évoquée, ainsi que dans une forte évolution des effectifs, pour renouveler les compétences et accélérer le programme de maintenance.

Enfin, des changements dans les paramètres de calcul expliquent l'augmentation restante : baisse de la production annuelle, changement de taux d'actualisation ${ }^{(b)}$ pour les dépenses futures de démantèlement et de gestion des déchets, changement de taux de rémunération du capital, inflation...

Ce cout de production n'est pas directement comparable aux couts de référence évalués dans différentes études, notamment de l'Agence de l'Environnement et de la Maîtrise de l'Energie (ADEME), sur les énergies renouvelables. En effet, ces derniers sont calculés pour un investisseur qui rentrerait aujourd'hui sur le marché avec de nouvelles centrales (qui auraient besoin d'être amorties financièrement, mais dont le cout de maintenance serait moindre) dont l'équivalent pour le nucléaire serait actuellement l'EPR, qui sera abordé plus loin. De la même manière, ce niveau de cout de production ne peut servir à dicter le choix entre la poursuite de l'exploitation en prolongeant la durée de vie des centrales ou leur remplacement à court terme par des centrales plus modernes, voire par d'autres sources d'énergie ${ }^{(c)}$.

\section{Sensibilité aux couts d'exploitation et d'investissements}

Le cout de production du nucléaire tel qu'indiqué plus haut est ce qu'on appelle le " cout courant économique ». Il inclut les dépenses d'exploitation, les investissements de maintenance, les provisions pour couvrir les dépenses futures (démantèlement et gestion des déchets et du combustible usé). Il inclut aussi un loyer économique tenant compte des investissements initiaux et de leur rémunération sur la totalité de la durée de fonctionnement envisagée. Ce cout est dominé aux trois quarts par les dépenses d'exploitation et les couts d'utilisation des actifs nucléaires (respectivement $42 \%$ et $35 \%$, voir figure 1). Ce cout courant économique exclut en revanche la recherche et développement, ainsi que la sureté/sécurité, financés sur fonds publics. Par ailleurs, il ne tient pas compte de l'historique du parc, et du fait que les investissements initiaux ont déjà largement été amortis. Ce cout de production differe du cout réel actuel d'EDF qui est moindre, et qui doit être couvert par les tarifs.
Le cout de production est très sensible à l'évolution des dépenses d'exploitation et des investissements de maintenance (16\%). Même si le projet industriel d'EDF repose sur l'hypothèse de " charges d'exploitation maitrisées ", les charges d'exploitation devraient progresser de 1,4\%/an en euros constants d'ici à 2025. Les investissements de maintenance, quant à eux, devraient continuer à augmenter pour atteindre un niveau moyen, supérieur de $16 \%$ au niveau d'investissement pris en compte dans le cout de 2013. Ce niveau d'investissements de maintenance ne se justifie toutefois que dans une perspective de l'allongement de la durée de vie des centrales. Ainsi, si les décisions politiques rendaient impossible ou trop incertaine cette prolongation, EDF devrait réviser son projet industriel : en effet, il parait économiquement irrationnel d'engager de très fortes rénovations de gros composants autour de 30/35 ans de durée de vie, si la durée restante de fonctionnement n'excède pas dix ans. De même, réaliser des investissements visant à élever le niveau de sureté à celui de la $3^{\mathrm{e}}$ génération n'aurait alors pas de sens.

En revanche, en raison de l'actualisation, les calculs sont peu sensibles aux évolutions des charges futures provisionnées. De cette manière, les incertitudes qui pèsent actuellement sur l'évaluation de ces charges n'ont en réalité qu'un impact très faible sur le cout de production, tel qu'il est calculé par la Cour. Une baisse (ou inversement une hausse) du taux d'actualisation conduit à faire évoluer le cout de production de $+0,8 \%$ (ou $-0,6 \%$ ). Si le devis de démantèlement augmentait de $50 \%$, le cout de production progresserait seulement de $2,5 \%$.

\section{L'impact de l'allongement de la durée de vie des centrales sur le cout de production}

La durée de fonctionnement des centrales est une hypothèse stratégique. Si le cout courant économique, et donc celui de production tel qu'il est calculé par la Cour, n'est pas très sensible à la durée d'exploitation des installations, cette durée est déterminante dans l'appréciation de la rentabilité des actifs nucléaires.

L'effet sur les couts de la prolongation de la durée de fonctionnement des centrales ne peut pas être mesuré à partir d'un simple calcul de sensibilité. En effet, cette 
prolongation aurait plusieurs impacts sur le cout de production :

- un impact à la baisse sur le loyer économique (impact cependant limité à 2 ou $3 \%$ pour un allongement de dix ans de la durée d'exploitation) ;

- un impact à la hausse sur les investissements de maintenance nécessaires à cette prolongation ;

- un impact à la baisse sur les provisions des dépenses futures compte tenu du décalage du calendrier de démantèlement.

En tenant compte de ces différents éléments et des hypothèses de hausse des dépenses d'exploitation citées plus haut la Cour des comptes a estimé le cout moyen de production pour la période 2011-2025 pour une durée de vie de 50 ans à 61,6 €/MWh. En cas de non prolongation de la durée de vie des centrales, il existe alors une incertitude sur le niveau d'investissements de maintenance à retenir, et les calculs du cout moyen de production sur la même période deviennent très incertains. EDF serait d'ailleurs peut-être amenée à conclure qu'il est, dans ce cas, économiquement rentable de fermer des centrales avant leurs 40 ans afin de ne pas engager de rénovations trop lourdes qui ne pourraient être amorties.

Il convient par ailleurs de noter que ce cout fait l'hypothèse que l'ensemble du parc sera prolongé au-delà de 40 ans pour une durée de 10 ans, alors qu'il est plus probable que les décisions seront plus hétérogènes (certains réacteurs fermant à 40 ans et d'autres étant prolongés jusqu'à 60 ans), compte tenu des différences de performance entre les différents réacteurs et pour répondre aux enjeux politiques de diversification du mix énergétique.

\section{L'incertitude du cout du nouveau nucléaire}

Quelle que soit leur durée de fonctionnement, les réacteurs actuels ne pourront être remplacés, à terme, que par des réacteurs de " $3^{\mathrm{e}}$ génération ", dont les conditions de sécurité sont supérieures à celles des réacteurs actuels. Les couts de production futurs à moyen/long terme de l'électricité nucléaire seront donc ceux de l'EPR, qui sont difficiles à appréhender finement aujourd'hui. L'EPR de Flamanville ne peut en effet servir de base à un calcul de cout de production moyen de l'EPR. Ce chantier, qui subit d'importants délais et surcouts, pâtit en effet de l'effet " tête de série » et de la remise en ordre de marche de la filière qui a perdu l'habitude de construire des réacteurs sur le sol français.

Toutefois, au regard de l'importance des couts de construction par rapport à ceux des réacteurs de $2^{\mathrm{e}}$ génération, et même si les EPR sont censés avoir des couts de fonctionnement moindres, il est probable que les couts de production seront sensiblement supérieurs à ceux du parc actuel. C'est la conclusion que l'on peut également tirer de l'accord passé en octobre 2013 entre EDF et le gouvernement britannique, avec un prix de vente de 92,5£/MWh (environ $106 € / \mathrm{MWh}$ ), même s'il y a de nombreuses différences entre l'EPR de Flamanville et ceux d'Hinkley Point (spécificité du site, normes britanniques, entreposage des déchets, prix du terrain...) et que le calcul du prix est sensible au choix fait pour le taux d'actualisation.

On peut également, pour avoir une idée de l'ordre de grandeur de ces couts futurs, regarder les hypothèses faites notamment par l'ADEME dans l'établissement de différents scénarios de mix énergétique à l'horizon 2050 : le cout de production du nouveau nucléaire est alors estimé à $80 € / \mathrm{MWh}$.

\section{Le cout d'un éventuel accident}

Un système international d'assurance $\mathrm{du}$ risque s'est mis en place, obligeant chaque réacteur à être assuré à hauteur de 750 millions d'euros et les États à prendre la relève jusqu'à 1,5 milliard. De son côté, la Cour des comptes a tenté, avec prudence, d'extrapoler le retour d'expérience de Fukushima. L'ordre de grandeur retenu pour le cout total d'un accident qui surviendrait en France est estimé entre 120 et 585 milliards d'euros. Cette fourchette est largement basée sur les travaux de l'Institut de Radioprotection et de Sûreté Nucléaire (IRSN) [3] qui visent à inclure tous les types de couts, même ceux que l'on ne sait pas chiffrer avec précision et indépendamment de ce qui est ou non indemnisable : depuis la réhabilitation du site et le contrôle radiologique, jusqu'aux effets sanitaires et psychologiques, en passant par la modification de la production d'électricité, ainsi que les conséquences en termes d'image sur le tourisme, l'activité agricole et les exportations.
En résumé, si on essaie de tirer une idée directrice de ces calculs à paramètres multiples, les investissements lourds de rénovation ne peuvent être viables que dans la perspective d'une durée de fonctionnement suffisamment longue. Si on prolonge les centrales anciennes, il faut s'attendre à une augmentation de 40-50\% du cout de production de l'électricité ; et plus ou moins du même ordre avec les centrales EPR. Si les incertitudes liées au cout du démantèlement ont peu d'impact sur le cout global, il y a des incertitudes bien plus importantes liées aux conditions financières, à la décision éventuelle de ne pas prolonger les centrales, et surtout au risque d'accident. Enfin, la Cour des comptes rappelle que le cout n'est pas le seul critère de prise de décision, et que de nombreux indicateurs pertinents pour les comparaisons ne sont simplement pas quantifiables sous forme financière.

\section{Références}

1. Cour des comptes, Les coûts de la filière électronucléaire, La Documentation française, 31 janvier 2012, www.ccomptes.fr/fr/publications/ les-couts-de-la-filiere-electro-nucleaire

2. Cour des comptes, Le coût de production de l'électricité nucléaire, Communication à la commission d'enquête de l'Assemblée nationale, Actualisation, 27 mai 2014,

www.ccomptes.fr/fr/publications/ le-cout-de-production-de-lelectricitenucleaire-actualisation-2014

3. IRSN, Coût économique des accidents nucléaires, avril 2013.

Estimation des coûts d'accidents nucléaires en France : Méthodologie appliquée par I'IRSN, 14 avril 2014 www.irsn.fr/FR/Actualites_presse/ Actualites/Pages/20140414_Estimationcouts-accidents-nucleaires-FranceMethodologie-IRSN.aspx

a. Rappelons que $50 € / \mathrm{MWh}$ équivaut à $5 \mathrm{ct} / \mathrm{kWh}$ à comparer au prix public de vente de l'électricité qui se situe vers 13 à $17 \mathrm{ct} / \mathrm{kWh}$ en 2018. Et un cout similaire si on achète la même quantité d'énergie sous forme de carburant à la pompe à essence.

b. Le taux d'actualisation est un paramètre qui permet l'aide à la décision, en comparant des couts actuels et futurs. Une plus grande importance accordée aux générations futures entraine une augmentation des couts prévus.

c.Voir dans ce dossier l'article de S. Huet (p. 41). 